\title{
Análisis de la variabilidad del desplazamiento de futbolistas de élite durante una temporada competitiva a partir de un modelo lineal mixto generalizado
}

\author{
Analysis of the variability of the movement of elite soccer players \\ during a competitive season of a generalized linear mixed model
}

\section{Análise da variabilidade do movimento de jogadores de futebol de elite durante uma temporada competitiva de um modelo linear generalizado misto}

\author{
Julen Castellano ${ }^{1 *}$ y Angel Blanco-Villaseñor ${ }^{2}$
}

${ }^{1}$ Universidad del País Vasco (UPVIEHU), ${ }^{2}$ Universidad de Barcelona

Resumen: Este estudio pretende describir la variabilidad en la distancia recorrida por futbolistas de élite a lo largo de una temporada. Se han analizado las distancias medias recorridas en diferentes rangos de intensidad por los jugadores de un mismo equipo de la segunda división espańola (Liga Adelante) durante la temporada 2013-14. La distancia total (DT) y metros por minuto $\left(\mathrm{m} \cdot \mathrm{min}^{-1}\right)$ recorrida por los futbolistas fueron registrados utilizando el sistema computerizado TRACAB system by ChyronHego a 25 frames por segundo, distinguiéndose cinco tipos de intensidades: paradoandando $\left(0-1 \mathrm{Km} \cdot \mathrm{h}^{-1}\right)$, marcha $\left(<7 \mathrm{Km} \cdot \mathrm{h}^{-1}\right)$, trote $\left(<14 \mathrm{Km} \cdot \mathrm{h}^{-1}\right)$, carrera $(<21$ $\left.\mathrm{Km} \cdot \mathrm{h}^{-1}\right)$ y sprint $\left(>21 \mathrm{Km} \cdot \mathrm{h}^{-1}\right)$. Después de un primer análisis descriptivo, a los datos se les aplicó un análisis lineal mixto generalizado (Linear Mixed). Los resultados mostraron que las distancias recorridas en los rangos de intensidad por encima de $7 \mathrm{Km} \cdot \mathrm{h}^{-1} \mathrm{y}$ por debajo de $21 \mathrm{Km} \cdot \mathrm{h}^{-1}$ variaron más que el resto durante la temporada. Esta variabilidad pudo deberse a variables situacionales como: lugar, nivel de los rivales y marcador final. Los resultados de estos análisis podrían tener una doble aplicación. Por un lado, el rendimiento físico de competición no parece ser la mejor manera de conocer el estado de forma del equipo, debido a la variabilidad contextual propia de la competición; siendo, en todo caso, los rangos de intensidad más elevados o los indicadores globales de rendimiento físico (DT y m·min-1) los más adecuados para valorarlo. Por otro, el seguimiento del rendimiento físico puede ser interesante considerarlo para adecuar estrategias de intervención en el proceso de entrenamiento con el objetivo de optimizar el rendimiento físico de los jugadores aplicado a contextos particulares de los partidos.

Palabras clave: Deportes colectivos, modelo lineal mixto, variables situacionales, demanda física

Summary: This study aims at describing the variability in the traveled distance for professional soccer players through a season. Average traveled distances have been analyzed in different ranks of intensity for players of a same team in the spanish second division (Liga Adelante) during the 2013-2014 season. The total distance traveled (DT) and meters per minute $\left(\mathrm{m} \cdot \mathrm{min}^{-1}\right)$ were recorded using the computerized system TRACAB ${ }^{\circ}$ system by ChyronHego at 25 frames per second, dinstinguishing between five kinds of intensity: still-moving $\left(0-1 \mathrm{Km} \cdot \mathrm{h}^{-1}\right)$, marching $\left(<7 \mathrm{Km} \cdot \mathrm{h}^{-1}\right)$, trotting $(<14$ $\left.\mathrm{Km} \cdot \mathrm{h}^{-1}\right)$, running $\left(<21 \mathrm{Km} \cdot \mathrm{h}^{-1}\right)$ and sprinting $\left(>21 \mathrm{Km} \cdot \mathrm{h}^{-1}\right)$. After an initial descriptive analysis, a linear mixed generalized one was applied to the data.

Dirección para correspondencia [Correspodence address]: Julen Castellano. Universidad del País Vasco (UPV/EHU). Facultad de Ciencias de la Actividad Física y el Deporte. C/ Portal de Lasarte 71. 01007 VitoriaGasteiz (Espańa). E-mail: julen.castellano@ehu.es
The results showed that traveled distances in ranks of intensity over $7 \mathrm{Km} \cdot \mathrm{h}$ ${ }^{1}$ and under $21 \mathrm{Km} \cdot \mathrm{h}^{-1}$ varied more than the others during the season. This variability could be due to situational variables such as: place, rivals' level and final score. The results of this analysis could have a double application. On one hand, the physical performance of the competition doesn't seem to be the best way to learn about the team's form, due to the contextual variability of the competition; in any case, the highest ranks of intensity or the global indicators of physical performance (DT and m-min-1) are the most adequate to enhance it. On the other hand, monitoring physical performance can be interesting if considered to improve intervention strategies during the training process with the objective to optimize the players' physical performance applied to particular contexts of the matches.

Resumo: Este estudo pretende descrever a variabilidade na distância percorrida por futebolistas de elite ao longo de uma época desportiva. Foram analisadas as distâncias médias percorridas em diferentes séries de intensidade pelos jogadores de uma mesma equipa na segunda divisão espanhola (Liga Adelante) durante a época desportiva 2013-14. A distância total (DT) e metros por minuto $(\mathrm{m} \cdot \mathrm{min}-1)$ percorridos pelos futebolistas foram recolhidas utilizando o sistema computorizado TRACAB ${ }^{\circ}$ system by ChyronHego a 25 frames por segundo, distinguindo-se cinco tipos de intensidades: parado-andando $\left(0-1 \mathrm{Km} \bullet \mathrm{h}^{-1}\right)$, marcha $\left(<7 \mathrm{~km} \bullet \mathrm{h}^{-1}\right)$, trote $\left(<14 \mathrm{~km} \bullet \mathrm{h}^{-1}\right)$, corrida $\left(<21 \mathrm{~km} \bullet \mathrm{h}^{-1}\right)$ e Sprint $\left(>21 \mathrm{~km} \bullet \mathrm{h}^{-1}\right)$. Após realizar uma análise descritiva, foi aplicada, aos dados, uma análise utilizando o modelo linear generalizado (Linear Mixed). Os resultados mostraram que as distâncias percorridas em séries de intensidade acima de $7 \mathrm{Km} \cdot \mathrm{h}^{-1}$ e abaixo de $21 \mathrm{Km} \cdot \mathrm{h}$ ${ }^{1}$ variaram mais do que o resto durante a época. Esta variabilidade pode dever-se a variáveis contextuais como: local, nível dos adversários e resultado do marcador final. Os resultados destas análises podem ter uma dupla aplicaçáo. Por um lado, o rendimento físico de competição não parece ser a melhor forma de conhecer o estado da forma da equipa, devido à variabilidade contextual própria da competiçấo, sendo que em todo o caso, as séries de intensidade mais elevada ou os indicadores globais de rendimento físico (DT e m-min-1) os mais adequados para avaliar o desempenho físico geral. Por outro lado, poderá ser interessante considerar a monitorização do desempenho físico para adequar estratégias de intervençáo no processo de treino com o objetivo de otimizar o rendimento físico dos jogadores aplicado a contextos específicos do jogo.

Palavras-chave: Desportos coletivos, modelo linear misto, variáveis contextuais, desempenho físico. 


\section{Introducción}

En el fútbol de rendimiento valorar las demandas de la competición (Carling, Bloomfield, Nelsen y Reilly, 2008), facilitado por la innovación tecnológica, ha sido foco de atención de multitud de trabajos (Castellano, Álvarez-Pastor, y Bradley, 2014), con los objetivos de conocer, entre otros, qué diferencia a los buenos de los que no lo son (Castellano, Casamichana y Lago, 2012), cómo va evolucionando el juego (Barreira, Garganta, Castellano, Prudente y Anguera, 2014) o para desarrollar contenidos de entrenamiento cada vez más específicos (Di Salvo, Baron, Tschan, Calderón Montero, Bachl, y Pigozzi, 2007). Algunas veces incluso, rangos altos de intensidad de desplazamiento han sido tomados como indicadores de rendimiento para valorar el estado de forma física de los jugadores (Mohr, Krustrup y Bangsbo, 2003) o valorar la posible fatiga entre primeras y segundas partes (Rampinini, Impellizzeri, Castagna, Coutts y Wisloff, 2009). Sin embargo, cada vez son más los estudios (Castellano, Blanco-Villaseñor y Álvarez-Pastor, 2011) que apuntan a que existen variables internas y externas al juego que ejercen una importante influencia en el rendimiento físico de los jugadores.

En este sentido, se está avanzando en el conocimiento de cómo la nacionalidad de los equipos (Casamichana y Castellano, 2014; Rienzi, Drust, Reilly, Carter y Martin, 2000), los estilos de juego particulares de las diferentes ligas europeas (Bradley et al., 2009; Dellal et al., 2011), el nivel de pericia técnico-táctica (Bradley, Di Mascio, Peart, Olsen, y Sheldon, 2010; Bradley et al., 2013), entre otros, hace que la respuesta física que los jugadores y equipos proponen en el desarrollo de la competición involucran diferentes niveles de exigencia. Es por esto que cuando se pretenda valorar la variabilidad de las demandas físicas de los jugadores, debería respetarse la homogeneidad de los mismos, equipos y ligas estudiadas, aunque esto no resulte sencillo de llevar a la práctica (Carling, Wright, Nelson y Bradley, 2014).

Pero, además de la particularidad en el 'uso' de las demandas físicas, a nivel interno, la dinámica del partido, también proporciona una variabilidad (Mohr, Krustrup y Bangsbo, 2003) que ha sido abordada en algunos trabajos (Gregson, Drust, Atkinson, y Salvo, 2010; Morgans, Adams, Mullen y Williams, 2014; Rampinini, Coutts, Castagna, Sassi y Imperizelli, 2007). Motivado, probablemente, al prolongado periodo competitivo de un campeonato de liga, la variabilidad del rendimiento físico asociado al momento o periodo de la temporada podría ser interesante de valorar para conocer si de entre las variables que contextualizan el rendimiento físico de los jugadores, la duración del campeonato responde a algún patrón determinado (Gregson et al., 2010; Morgans et al., 2014; Rampinini et al, 2007). Para la liga inglesa se sabe que la variabilidad entre partidos, expresado a partir de coeficiente de variación $(\mathrm{CV})$ puede estar en torno al 16\%, pu- diendo llegar incluso al 31\% cuando las variables analizadas se refieren a las distancias recorridas a velocidades de carrera alta (Gregson et al., 2010). Valores similares de variabilidad, con unos CV entre el 9\% y el 25\%, dieron los jugadores italianos analizados por Mohr et al. (2003), comprobando que fue en la parte final de la temporada cuando los jugadores mostraron un mayor nivel de rendimiento físico. Coincidiendo también Rampinini et al. (2007) en los valores del CV y en la mejora del rendimiento físico a lo largo de la temporada. Por otro lado, en un estudio realizado con seis jugadores ingleses (Morgans et al., 2014) se encontró una reducción en la distancia recorrida a altas velocidades y sprint en la mitad y al final de la temporada respecto al primer tercio de la misma, siendo la fatiga una posible causa que lo justificase. Sin embargo, estos resultados podrían estar afectados por variables como el lugar de disputa de los partidos (casa/fuera), primeras y segundas partes, el nivel del oponente o el marcador final. Se desconoce además, si la variabilidad podría estar condicionada por el nivel de los jugadores, siendo más regulares en el rendimiento físico cuando se trata de jugadores de mayor nivel competitivo.

En esta línea, estudios paralelos pretenden explicar el porqué de esta variabilidad asociada a la demanda física. Variables como el sistema de juego empleado por el equipo (Bradley et al., 2011) o el de los rivales (Carling, 2011), el porcentaje de posesión del balón (Bradley, Lago, Rey y Gómez, 2013; Gregson et al., 2010), el tiempo de juego efectivo (Castellano, Blanco-Villaseñor y Álvarez-Pastor, 2011) o el resultado (O’Donoghue y Tenga, 2001), son algunos de los estudiados. A diferencia de los modelos tradicionales, pocos autores (Castellano, Blanco-Villaseñor y Álvarez-Pastor, 2011) han utilizado datos longitudinales para analizar las múltiples medidas como un solo resultado multivariante. Una de las razones que justifica este segundo tipo de análisis, Linear Mixto Generalizado, es debido a que en la investigación en Ciencias Sociales y del Comportamiento comúnmente presenta situaciones en las que es difícil utilizar los modelos lineales clásicos de análisis de varianza y regresión porque no se cumplen los supuestos de independencia, normalidad, igualdad de varianzas o incluso linealidad. La modelación de datos científicos en el marco teórico de los modelos lineales y generalizados mixtos brinda la posibilidad de analizar datos con estructuras de dependencia, homocedasticidad y falta de normalidad. Es así posible relajar los supuestos tradicionales del modelo lineal general y modelar, de manera flexible, estructuras complicadas de datos.

Por todo lo comentado anteriormente, para valorar la posible existencia de cambios en el rendimiento físico de los jugadores a lo largo de una misma temporada, el presente trabajo tiene como objetivo estudiar las distancias recorridas 
por los jugadores en diferentes rangos de intensidad a lo largo de los 42 partidos disputados, habiendo sido estos agrupados en diferentes momentos o periodos a lo largo de la temporada. Los resultados obtenidos podrían ser utilizados para valorar cómo evoluciona el rendimiento físico de los jugadores y, por tanto, disponer de información para proponer estrategias de intervención específicas de acuerdo a los objetivos marcados por el cuerpo técnico durante la temporada respecto a cuestiones como: estrategias de planificación de cara al entrenamiento para la adquisición de la forma deportiva, rotación de jugadores, propuesta de tareas específicas de entrenamiento o tipo de entrenamiento compensatorio de jugadores menos habituales.

\section{Método}

\section{Participantes}

En este estudio han sido analizados 19 jugadores de campo (excluidos los porteros) del Deportivo Alavés (ALA) de la segunda división española de fútbol (Liga Adelante) durante la temporada 2013-14. Hubo disponibilidad de conocer las demandas físicas de carrera en 33 de los 42 partidos disputados por el equipo, lo que han supuesto un total de 554 observaciones individuales, debido a que solo se incluyeron aquellos registros de jugadores que finalizaron la parte completa del partido. Además, aquellos jugadores que no completaron un mínimo de 12 registros durante la temporada fueron excluidos. Para clasificar a los jugadores en diferentes demarcaciones se empleó una categorización previamente utilizada (Andrzejewski, Chmura, y Pluta, 2014; Bradley, Lago-Peñas y Rey, 2014): defensas laterales (DL, $n=4)$, defensas centrales (DC, $\mathrm{n}=5$ ), medios centros (MC, $\mathrm{n}=5$ ), medios laterales (ML, $\mathrm{n}=3$ ) y delanteros (DEL, $\mathrm{n}=2$ ). El número de registros por jugador varió desde 12 a 60, siendo la media 26 partes completas analizadas por jugador.

Tabla 1. Número de registros utilizados (n) y clasificados por las variables: lugar, parte y demarcación.

\begin{tabular}{lrrrrrrr}
\hline \multicolumn{7}{c}{ Demarcación } \\
\hline Lugar & Parte & DC & DL & MC & ML & DE & Total \\
\hline \multirow{2}{*}{ Casa } & $1^{\text {a }}$ & 38 & 24 & 45 & 29 & 25 & 161 \\
& $2^{\text {a }}$ & 41 & 9 & 40 & 20 & 18 & 128 \\
\multirow{4}{*}{ Fuera } & $1^{\text {a }}$ & 32 & 19 & 40 & 32 & 25 & 148 \\
& $2^{\text {a }}$ & 30 & 10 & 38 & 19 & 20 & 117 \\
& Total & 141 & 62 & 163 & 100 & 88 & 554
\end{tabular}

Nota: DC es defensa central, DL es defensa lateral, MC es medio centro, ML es medio lateral y DE es delantero y media punta.

Los datos han sido tratados de acuerdo con la Declaración de Helsinki. Fueron disociados previo a la realización de los análisis de aquellos datos personales que pudieran identificarles. Además se tuvo consentimiento del club implicado y se recibió dictamen favorable del comité de ética con seres humanos (CEISH) de la Universidad del País Vasco (UPV/ EHU).

\section{Variables}

Variables de clasificación

Como variables de clasificación, al igual que en trabajos anteriores (Castellano, Blanco-Villaseñor y Álvarez-Pastor, 2011) se consideraron las siguientes: nivel rival, momento, lugar, marcador final y parte. Para establecer el nivel de los equipos rivales, similar a lo propuesto en anteriores trabajos (Lago, 2009; Rampinini, Impellizzeri, Castagna, Coutts, y Wisloff, 2009; Taylor, Mellalieu, James, y Shearer, 2008) se agruparon los equipos en tres niveles, siendo los siete primeros clasificados al final de liga el nivel 1 (del $1^{\text {er }}$ clasificado hasta el 7o), los seis siguientes el nivel 2 (desde el $8^{\circ}$ al $14^{\circ}$ ) y el restante (del $15^{\circ}$ hasta $22^{\circ}$ ) el nivel 3. El Alavés esa temporada acabó clasificado en $18^{\circ}$ lugar, es decir, perteneció al nivel 3. Para codificar la variable momento, a diferencia de trabajos anteriores donde se utilizaron únicamente tres periodos (Mohr et al., 2003; Rampinini et al., 2007), se agruparon los partidos en siete periodos, cada uno de los cuales aglutinó seis partidos de competición ( 7 periodos $* 6$ partidos $=42$ jornadas). En la variable lugar se distinguieron dos niveles, casa o fuera, por lo que se diferenció al Alavés cuando jugó como local o visitante. El marcador final tuvo también tres niveles, ganar, empatar o perder, tal y como lo han propuesto otros autores (O’Donoghue y Tenga, 2001). Finalmente la variable parte diferenció las dos partes que componen el partido, primera y segunda parte.

Categorías de movimiento y rangos de intensidad

En línea con trabajos anteriores (Castellano, Blanco-Villaseñor y Álvarez-Pastor, 2011; Mohr et al., 2003; Rampinini et al., 2007) además de la distancia total recorrida (DT), las demandas físicas de los jugadores fueron analizados a partir de cinco rangos de intensidad de desplazamiento: DT1 o parado $\left(<1 \mathrm{Km} \cdot \mathrm{h}^{-1}\right)$, DT $<7$ o trote $\left(<7 \mathrm{Km} \cdot \mathrm{h}^{-1}\right)$, DT $<14$ o carrera $\left(<14 \mathrm{Km} \cdot \mathrm{h}^{-1}\right)$, DT<21 o carrera rápida $\left(<21 \mathrm{Km} \cdot \mathrm{h}^{-1}\right)$ y DT>21 o sprint $\left(>21 \mathrm{Km} \cdot \mathrm{h}^{-1}\right)$. Además, se emplearon las velocidades de distancia de carrera recorrida por minuto de partido $\left(\mathrm{m} \cdot \min _{\mathrm{TT}}\right)$.

\section{Procedimiento}

Con el sistema de videotracking mediante la aplicación TRA$C A B^{\circ}$ se obtuvieron los informes de los desplazamientos de 
los jugadores en cada uno de sus partidos, que es gestionado desde la aplicación Mediacoach ${ }^{\oplus}$. La fiabilidad y validez de este tipo de sistemas ha sido evaluado anteriormente $(\mathrm{Bu}-$ chheit et al., 2014; Di Salvo, Collins, Macneill, y Cardinale, 2006).

\section{Análisis de datos}

Primeramente se realizó un análisis descriptivo de los datos, para lo cual fue utilizado el paquete estadístico SPSS versión Windows 20. Para una mejor interpretación porcentual del grado de variabilidad se calculó el coeficiente de variación $(\mathrm{CV})$ en \%. En todos los análisis estadísticos fue admitido un nivel de significación de $p<0,05$.

En segundo lugar, fue aplicado un Modelo Lineal Mixto generalizado a partir del SAS para Windows 9.1 (SAS Institute Inc. SAS/STAT, 1999) con el objetivo de analizar la dependencia de los cambios con variables como resultado, lugar, momento la temporada, nivel del rival y parte. Los modelos estadísticos mixtos permiten modelar la respuesta de un estudio experimental u observacional como función de factores o covariables cuyos efectos pueden considerarse tanto como constantes fijas o variables aleatorias. Cada modelo estadístico que contiene una media general, $\mu$, es un modelo mixto por definición, ya que también contiene un término de error aleatorio, y por tanto contiene ambos tipos de efectos. Sin embargo, en la práctica, el nombre modelo mixto se reserva usualmente para cualquier modelo que contiene efectos fijos distintos a $\mu$ y efectos aleatorios diferentes a los errores aleatorios. En general, un efecto es considerado como fijo si los niveles del factor asociado han sido arbitrariamente determinados por el investigador, tal y como ocurre en este estudio que presentamos sobre la variabilidad física. De esta manera con el Modelo Mixto implementado podemos cuantificar la relación entre variables dependientes continuas (distancias recorridas en diferentes rangos de intensidad) y varias variables predictoras, anteriormente comentadas.

\section{Resultados}

En la figura 1 se recoge la DT media recorrida (en un aparte de un partido) por los jugadores en cada uno de los siete periodos en los que se dividió la temporada competitiva. Como puede observarse, sobretodo, fue en el primer periodo donde el equipo recorrió de manera significativa una menor distancia total respecto al resto de los periodos a excepción del séptimo.

Figura 1. Distancia total (DT) recorrida por jugador en una parte (en metros) en los siete periodos de la temporada.

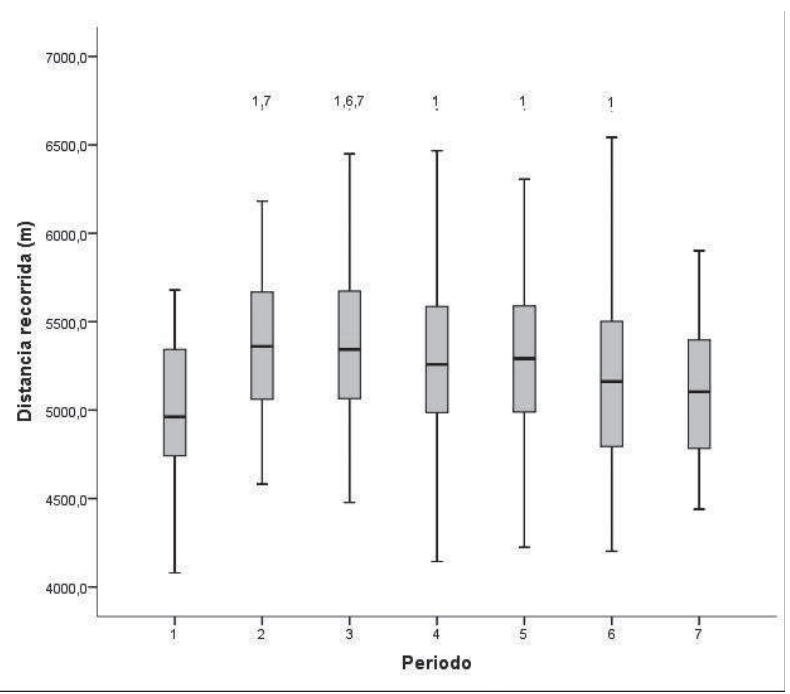

Nota: Los siete periodos abarcan cada uno de ellos 6 partidos de liga, hasta completar los 42 que componen la temporada, siendo 1 el periodo que abarcan los 6 primeros partidos, sucesivamente hasta el $7^{\circ}$ que engloban los 6 últimos. 1 es significativamente superior al periodo 1,6 es significativamente superior al periodo 6 y 7 es significativamente superior al periodo 7 , para $\mathrm{p}<0.05$.

En la tabla 2 se recogen los valores medios y coeficientes de variación de las diferentes variables físicas analizadas distinguiéndose el jugador. Como puede observarse, las distancias recorridas en la categoría de parado fue en la que mayor $\mathrm{CV}$ se estimó, incrementándose a medida que el rango de velocidad fue aumentando. Finamente, fueron las variables DT, y $\mathrm{m} \cdot \min _{\mathrm{TT}}$.

Por otra parte, todas las demarcaciones tuvieron una variabilidad similar, aumentando a medida que se incrementó la velocidad de carrera, también para DT1. Para todas ellas también fueron las variables DT y $\mathrm{m} \cdot \mathrm{min}_{\mathrm{TT}}$ las que menos variaron, siendo el CV para los DC $=70 \%, 7 \%, 11 \%, 21 \%$ y $42 \%$, para $\mathrm{DL}=92 \%, 7 \%, 10 \%, 17 \%$ y $29 \%$, para $\mathrm{MC}$ $=67 \%, 8 \%, 10 \%, 17 \%$ y $47 \%$, para $\mathrm{ML}=74 \%, 7 \%, 12 \%$, $19 \%$ y $30 \%$ y para $\mathrm{DE}=93 \%, 9 \%, 12 \%, 19 \%$ y $33 \%$ para los rangos de carrera de DT1, DT7, DT14, DT<21 y DT $>21$, respectivamente. 
Tabla 2. Medias (X) y coeficiente de variación (CV) en metros para cada una de las variables físicas analizadas.

\begin{tabular}{|c|c|c|c|c|c|c|c|c|c|c|c|c|c|c|c|c|}
\hline \multirow[b]{2}{*}{ demarcación } & \multirow[b]{2}{*}{ jugador } & \multirow[b]{2}{*}{$\mathrm{n}$} & \multicolumn{2}{|c|}{ DT1 } & \multicolumn{2}{|c|}{ DT $<7$} & \multicolumn{2}{|c|}{$\mathrm{DT}<14$} & \multicolumn{2}{|c|}{$\mathrm{DT}<21$} & \multicolumn{2}{|c|}{$\mathrm{DT}>21$} & \multicolumn{2}{|l|}{ DT } & \multicolumn{2}{|c|}{$\mathrm{m} / \mathrm{min}_{\mathrm{TT}}$} \\
\hline & & & $\mathrm{X}$ & $\mathrm{CV}$ & $\mathrm{X}$ & $\mathrm{CV}$ & $\mathrm{X}$ & $\mathrm{CV}$ & $\mathrm{X}$ & $\mathrm{CV}$ & $\mathrm{X}$ & $\mathrm{CV}$ & $\mathrm{X}$ & $\mathrm{CV}$ & $\mathrm{X}$ & $\mathrm{CV}$ \\
\hline \multirow{5}{*}{$\mathrm{DC}$} & 1 & 27 & 247 & $90 \%$ & 1805 & $7 \%$ & 1939 & $11 \%$ & 1068 & $27 \%$ & 180 & $39 \%$ & 5238 & $9 \%$ & 111 & $10 \%$ \\
\hline & 2 & 20 & 174 & $60 \%$ & 1775 & $9 \%$ & 1959 & $12 \%$ & 749 & $21 \%$ & 154 & $40 \%$ & 4810 & $8 \%$ & 102 & $9 \%$ \\
\hline & 3 & 54 & 165 & $99 \%$ & 2017 & $7 \%$ & 1944 & $10 \%$ & 647 & $20 \%$ & 109 & $48 \%$ & 4883 & $5 \%$ & 104 & $5 \%$ \\
\hline & 4 & 12 & 135 & $62 \%$ & 1991 & $5 \%$ & 1728 & $15 \%$ & 522 & $19 \%$ & 101 & $43 \%$ & 4477 & $6 \%$ & 96 & $6 \%$ \\
\hline & 5 & 28 & 168 & $39 \%$ & 1804 & $6 \%$ & 1908 & $9 \%$ & 790 & $17 \%$ & 172 & $38 \%$ & 4841 & $6 \%$ & 104 & $6 \%$ \\
\hline \multirow{4}{*}{$\mathrm{DL}$} & 6 & 36 & 143 & $67 \%$ & 1796 & $6 \%$ & 1941 & $8 \%$ & 1120 & $15 \%$ & 419 & $28 \%$ & 5420 & $5 \%$ & 115 & $5 \%$ \\
\hline & 7 & 17 & 125 & $37 \%$ & 1614 & $8 \%$ & 2176 & $9 \%$ & 1059 & $21 \%$ & 273 & $20 \%$ & 5247 & $5 \%$ & 112 & $7 \%$ \\
\hline & 8 & 32 & 144 & $94 \%$ & 1818 & $6 \%$ & 1859 & $11 \%$ & 717 & $16 \%$ & 242 & $35 \%$ & 4780 & $6 \%$ & 102 & $7 \%$ \\
\hline & 9 & 36 & 184 & $168 \%$ & 1707 & $9 \%$ & 2271 & $11 \%$ & 1042 & $15 \%$ & 298 & $35 \%$ & 5501 & $6 \%$ & 117 & $6 \%$ \\
\hline \multirow{5}{*}{ MC } & 10 & 36 & 173 & $99 \%$ & 1509 & $7 \%$ & 2598 & $10 \%$ & 1444 & $18 \%$ & 242 & $38 \%$ & 5967 & $6 \%$ & 128 & $6 \%$ \\
\hline & 11 & 10 & 137 & $51 \%$ & 1738 & $6 \%$ & 2260 & $11 \%$ & 1520 & $14 \%$ & 233 & $45 \%$ & 5888 & $6 \%$ & 126 & $8 \%$ \\
\hline & 12 & 22 & 117 & $78 \%$ & 1628 & $8 \%$ & 2514 & $9 \%$ & 1122 & $18 \%$ & 117 & $70 \%$ & 5498 & $5 \%$ & 117 & $6 \%$ \\
\hline & 13 & 25 & 93 & $49 \%$ & 1620 & $9 \%$ & 2424 & $10 \%$ & 1299 & $17 \%$ & 142 & $36 \%$ & 5578 & $6 \%$ & 120 & $6 \%$ \\
\hline & 14 & 49 & 211 & $57 \%$ & 1658 & $9 \%$ & 2299 & $11 \%$ & 1113 & $21 \%$ & 171 & $46 \%$ & 5451 & $7 \%$ & 116 & $7 \%$ \\
\hline \multirow{3}{*}{ ML } & 15 & 26 & 135 & $98 \%$ & 1836 & $8 \%$ & 1792 & $14 \%$ & 894 & $20 \%$ & 282 & $28 \%$ & 4938 & $7 \%$ & 106 & $7 \%$ \\
\hline & 16 & 24 & 138 & $61 \%$ & 1879 & $5 \%$ & 2013 & $9 \%$ & 1011 & $12 \%$ & 259 & $25 \%$ & 5300 & $4 \%$ & 114 & $4 \%$ \\
\hline & 17 & 10 & 117 & $64 \%$ & 1726 & $7 \%$ & 2223 & $14 \%$ & 1260 & $24 \%$ & 339 & $38 \%$ & 5665 & $4 \%$ & 123 & $4 \%$ \\
\hline \multirow{3}{*}{$\mathrm{DE}$} & 18 & 60 & 195 & $84 \%$ & 1849 & $9 \%$ & 2185 & $11 \%$ & 820 & $21 \%$ & 219 & $35 \%$ & 5267 & $5 \%$ & 112 & $7 \%$ \\
\hline & 19 & 30 & 260 & $103 \%$ & 1991 & $9 \%$ & 1714 & $13 \%$ & 861 & $16 \%$ & 207 & $30 \%$ & 5034 & $8 \%$ & 107 & $9 \%$ \\
\hline & Total & 554 & 170 & $95 \%$ & 1786 & $11 \%$ & 2099 & $16 \%$ & 975 & $31 \%$ & 216 & $52 \%$ & 5246 & $9 \%$ & 112 & $9 \%$ \\
\hline
\end{tabular}

Nota: DC es defensa central, DL es defensa lateral, MC es medio centro, ML es medio lateral o interior, DE es delantero, DT es distancia total recorrida, DT1 o parado es a $\left.<1 \mathrm{Km} \cdot \mathrm{h}^{-1}\right), \mathrm{DT}<7$ es a trote $\left(<7 \mathrm{Km} \cdot \mathrm{h}^{-1}\right), \mathrm{DT}<14 \mathrm{o}$ a carrera $\left(<14 \mathrm{Km} \cdot \mathrm{h}^{-1}\right), \mathrm{DT}<21$ es a carrera rápida $\left(<21 \mathrm{Km} \cdot \mathrm{h}^{-1}\right), \mathrm{DT}>21 \mathrm{o}$ sprint $\left(>21 \mathrm{Km} \cdot \mathrm{h}^{-1}\right), \mathrm{m} / \mathrm{min}_{\mathrm{TT}}$ es metros recorridos en el tiempo total.

Respecto al análisis lineal mixto generalizado los resultados figuran en la tabla 3. Como puede observarse, las variables $\mathrm{DT}<14$ y DT<21 mostraron, de manera significativa, variabilidad en todas las variables situacionales estudiadas. Sin embargo, la distancia recorrida en las primeros rangos de velocidad (DT1 y DT<7) y la recorrida a sprint (DT>21) no mostraron estas diferencias. Respecto a DT existieron en todos los casos diferencias significativas a excepción de la variable lugar.

Tabla 3. Grado de significación $(\operatorname{Pr}>\mathrm{p})$ de las distancias recorridas a diferente rango de intensidad respecto a las variables situacionales estudiadas.

\begin{tabular}{llllll}
\hline Variable & Momento & Nivel del rival & Lugar & Marcador & Parte \\
\hline DT1 & $\mathrm{ns}$ & $\mathrm{ns}$ & $\mathrm{ns}$ & $\mathrm{ns}$ & $\mathrm{ns}$ \\
DT $<7$ & $\mathrm{~ns}$ & $\mathrm{~ns}$ & $\mathrm{~ns}$ & $\mathrm{~ns}$ & $\mathrm{~ns}$ \\
DT $<14$ & $* *$ & $* *$ & $* *$ & $* *$ & $* *$ \\
DT $<21$ & $* *$ & $* *$ & $* *$ & $* *$ & $* *$ \\
DT $>21$ & $\mathrm{~ns}$ & $\mathrm{~ns}$ & $\mathrm{~ns}$ & $\mathrm{~ns}$ & $\mathrm{~ns}$ \\
\hline DT & $* *$ & $*$ & $\mathrm{~ns}$ & $*$ & $*$ \\
\hline
\end{tabular}

Nota: ns es no significativo, ${ }^{*}$ es $\mathrm{p}<0.05 \mathrm{y}^{* *}$ es $\mathrm{p}<0.01$.

\section{Discusión}

El objetivo del presente estudio fue valorar la variabilidad en el rendimiento físico de los jugadores de un equipo de la segunda división española de fútbol (Liga Adelante) a lo largo de una misma temporada. Para tal fin se empleó la distancia recorrida en diferentes rangos de intensidad. Aunque previamente ya se ha estudiado la variabilidad asociada al rendimiento físico de los jugadores de élite (Gregson et al., 2010; Morgans et al., 2014; Rampininiet al., 2007), este es el primer trabajo que estudia dicho aspecto en un equipo de la segunda división, empleando para ello un modelo mixto de análisis con las que explicar cómo se vieron afectas las variables dependientes, distancias recorridas a diferente intensidad, por la presencia de variables independientes predictoras como el momento, resultado, parte, nivel del rival y lugar.

El primer aspecto que se puede destacar de los resultados de este estudio es que únicamente los periodos $1^{\circ}$ y $7^{\circ}$ variaron respecto a los otros cinco en la distancia recorrida por los jugadores en un parte del partido. La literatura no es conclu- 
yente en este aspecto, porque mientras Mohr et al. (2003) encontraron mejoras en la distancia recorrida por los jugadores a medida que avanzó la temporada, Morgans et al. (2014) registraron una reducción en la distancia recorrida en rangos altos de velocidades al final de la temporada competitiva respecto al inicio. Diferentes estrategias planteadas por los equipos en su planificación del entrenamiento o la existencia de periodos congestionados de competición (Dellal, Lago, Rey, Chamari, y Orhant, 2013) podrían ser la explicación de estas diferencias.

A pesar de la homogeneidad general del rendimiento físico mostrado por el equipo a lo largo del ańo, los resultados del presente estudio corroboran la variabilidad de las demandas físicas asociadas a la competición de manera individual. Tal y como se ha indicado en trabajos realizados con jugadores de máximo nivel (Gregson et al., 2010; Morgans et al., 2014; Rampininiet al., 2007), la realidad competitiva da pie a un amplio espectro de distancias recorridas por los mismos jugadores durante la temporada, con una variabilidad que puede oscilar hasta en más del $100 \%(\mathrm{CV})$, es decir, con una oscilación mayor a la propia media. Lo novedoso del presente estudio tiene que ver con el hecho de que los resultados desvelan una mayor variabilidad, por encima del 31\% estimado por Gregson et al. (2010) en los jugadores de segundo nivel con respecto a los de una división superior. La variabilidad es mayor a medida que aumenta la intensidad de carrera, llegando incluso a un valor medio para el total de las demarcaciones de más del 50\%. Sin embargo, un aspecto que podemos destacar es que en el rango DT1 es el intervalo donde mayor variabilidad presentó. Al tratarse de la actividad de parado/andando que habitualmente se realiza cuando el balón está parado podría ser que las diferentes duraciones de los partidos y, por tanto, la duración variada del tiempo de juego efectivo (Castellano, Blanco-Villaseñor y Álvarez-Pastor, 2011) en cada partido haya sido uno de los motivos. En cualquier caso se trata de una actividad de poco interés para analizar el rendimiento físico, ya que son momentos donde el jugador se toma tiempo para recuperar y recolocarse a la espera de que el juego vuelva a reanudarse.

Con respecto a la variabilidad de las demarcaciones se puede resaltar que en los rangos de intensidad que caracteriza el perfil físico de cada una de los puestos sobre el terreno de juego en el ámbito profesional (Di Salvo et al., 2007) fueron los que presentaron un menor grado de variabilidad. Así por ejemplo, las distancias recorridas por los interiores (ML) y delanteros (DE) a $>21 \mathrm{Km} \cdot \mathrm{h}^{-1}$ presentaron un menor valor en el CV. Por el contrario, demarcaciones como DL y MC tuvieron una menor variabilidad en las distancias recorridas a $<17$ $\mathrm{y}<21 \mathrm{Km} \cdot \mathrm{h}^{-1}$. Para todas las demarcaciones, cualquiera de los dos indicadores, DT, distancias recorrida por minuto en relación al tiempo total mostraron una reducida variabilidad $(\mathrm{CV}=4 \% / 10 \%)$.

A partir de la aplicación del análisis MIXED se estimó que fueron las variables DT1, DT<7 y sprint (DT>21) las que no se vieron afectadas sistemáticamente por las variables contextuales a partir de las cuales fueron analizadas. Por este motivo, cuando estudios previos aportan correlaciones positivas entre la distancia recorrida a alta intensidad y un resultado favorable en el partido (Bradley et al., 2010, Bradley et al., 2009) quizás, a pesar de la variabilidad intra-sujeto existente, cabe esperar cierta regularidad en el rendimiento físico independientemente de las variables contextuales que hacen 'único' cada partido. En este sentido, el nivel del rival, el resultado o la estrategia adoptada por el equipo (Currell y Jeukendrup, 2008) podrían no afectar precisamente a la distancia recorrida en esta intensidad de carrera. Más investigación al respecto sería necesaria.

Por otro lado, las distancias recorridas en rangos intermedios de velocidad, $<14$ y $<21 \mathrm{Km} \cdot \mathrm{h}^{-1}$, mostraron diferencias significativas en todas las variables predictoras estudiadas. Sin embargo, la variabilidad estimada en los diferentes periodos en los que fue dividida la temporada competitiva no mostraron una tendencia lineal de mejora o empeoramiento, con lo que se puede descartar fatiga residual del equipo (Cormack, Newton y McGuigan, 2008).

La conclusión general del estudio podría resumirse en que los jugadores desarrollaron un rendimiento físico de carrera a lo largo de la temporada con un elevado grado de variabilidad, debido a las variables consideradas en el presente estudio como el momento de la temporada, lugar donde se disputó el encuentro (casa o fuera), nivel del oponente o resultado del partido. Probablemente, esta inestabilidad detectada podría estar influenciada por otras variables no estudiadas como la forma de jugar, el sistema empleado (por el equipo y por el rival), el estado de forma de los jugadores, perfil individual de los jugadores que componen el equipo, expulsiones o lesiones (una vez consumados los tres cambios permitidos por el reglamento) que dejan al equipo en inferioridad o superioridad numérica, etc., constatándose que el uso de este tipo de indicadores físicos no deberían utilizarse de manera exclusiva y puntual para detectar estados de fatiga o valorar el rendimiento físico de los equipos. La variabilidad contextual propia de la competición hace difícil este tipo de valoraciones. Sin embargo, un seguimiento prolongado de jugadores y equipos puede dar 'pistas' con las que afinar la posible valoración de cómo evoluciona el rendimiento físico de los jugadores y, de si en contextos particulares de competición puede desplegarse el potencial físico requerido en el momento. Además, parece que la variabilidad de la distancia recorrida en los diferentes rangos de carrera puede estar condicionada por la demarcación ocupada por los jugadores; existiendo una menor variabilidad en aquellas intensidades de carreras que más definen un rol en el sistema del equipo. Finalmente, creemos que a partir de los resultados de este estudio, se está en condiciones de manejar información de interés con 
las que proponer estrategias de intervención específicas de acuerdo a los objetivos marcados por el cuerpo técnico durante la temporada respecto a rotación de jugadores, tareas de entrenamiento, entrenamiento compensatorio para jugadores menos habituales en el 'once inicial', entre otros. Reducir la variabilidad en la medida de lo posible e incidir en aquellos aspectos manejables por jugadores y el cuerpo técnico respecto a estrategias más o menos conservadoras de desgaste físico o cambios de ritmo durante el desarrollo del juego, con el objetivo de optimizar el rendimiento físico de los jugadores aplicado a contextos particulares de los partidos.
Agradecimientos: Este trabajo forma parte de dos proyectos de investigación, de ámbito estatal titulado Observación de la interacción en deporte y actividad física: Avances técnicos y metodológicos en registros automatizados cualitativos-cuantitativos [DEP2012-32124] subvencionado por la Secretaría de Estado de Investigación, Desarrollo e Innovación del Ministerio de Economia y Competitividad de Espańa, y autonómico titulado Evaluación del proceso de entrenamiento y la competición en el fútbol de formación [Código 13523] subvencionado por la Universidad del País Vasco (UPV/EHU).

\section{Referencias}

1. Andrzejewski, M., Chmura, J., y Pluta, B. (2014). Analysis of motor and technical activities of profesional soccer players of the UEFA Europa League. International Journal of Performance Analysis in Sport, 14, 504-523.

2. Barreira, D., Garganta, J., Castellano, J., Prudente, J. y Anguera, M. T. (2014). Evolución del ataque en el fútbol de élite entre 1982 y 2010: aplicación del análisis secuencial de retardos. Revista de Psicología del Deporte, 23(1), 139-146.

3. Bradley, P. S, Di Mascio M., Peart, D., Olsen, P., y Sheldon, B. (2010). High-intensity activity profiles of elite soccer players al different performance levels. Journal of Strength and Conditioning Research, 24(9), 2343-51.

4. Bradley, P. S., Carling, C., Archer, D., Roberts, J., Dodds, A., Di Mascio, M., Paul, D., Diaz, A. G., Peart, T., y Krustrup, P. (2011). The effect of playing formation on high-intensity running and technical profiles in English Premier League soccer matches. Journal of Sports Science, 29, 821-830.

5. Bradley, P. S., Carling, C., Gomez-Diaz, A., Hood, P., Barnes, C., Ade, J., Boddy, M., Krustrup, P., y Mohr, M. (2013). Match performance and physical capacity of players in the top three competitive standards of English professional soccer. Human Movement Science, 32(4), 80821 .

6. Bradley, P. S., Lago, C., Rey, E. y Gomez, A. (2013), The effect of high and low percentage ball possession on physical and technical profiles in English FA Premier League soccer matches. Journal of Sports Sciences, 31, 1261-1270.

7. Bradley, P.S., Sheldon, W., Wooster, B., Olsen, P., Boanas, P. y Krustrup, P. (2009). High intensity running in English FA Premier League soccer matches. Journal of Sports Sciences, 27, 159-168.

8. Buchheit, M., Allen, A., Poon, T. K., Modonutti, M., Gregson W., y Di Salvo, W. (2014). Integrating different tracking systems in football: multiple camera semi-automatic system, local position measurement and GPS technologies. Journal of Sports Sciences, DOI: $10.1080 / 02640414.2014 .942687$

9. Carling, C. (2011): Influence of opposition team formation on physi$\mathrm{cal}$ and skill-related performance in a professional soccer team, European Journal of Sport Science, 11(3), 155-164.

10. Carling, C., Bloomfield, J., Nelsen, L. y Reilly, T. (2008). The role of motion analysis in elite soccer: Contemporary performance measurement techniques and work rate data. Sports Medicine, 338, 839-862.

11. Carling, C., Wright, C., Nelson, L. N. y Bradley, P. (2014). Comment on performance analysis in football: a critical review and implications for future research. Journal of Sports Science.32(1):2-7.

12. Casamichana, D. y Castellano, J. (2014 in press). Variables contextuales y distancia recorrida en la copa mundial Sudáfrica'10. Revista Internacional de Medicina y Ciencias de la Actividad Física y el Deporte.
13. Castellano, J., Blanco-Villaseńor, A., y Álvarez-Pastor, D. (2011). Contextual Variables and Time-Motion Analysis in Soccer. International Journal of Sports Medicine, 32, 415-421.

14. Castellano, J., Álvarez-Pastor, D., y Bradley, P. S. (2014). Evaluation of Research Using Computerised Tracking Systems (Amisco ${ }^{\circ}$ and Prozone $^{\odot}$ ) to Analyse Physical Performance in Elite Soccer: A Systematic Review. Sports Medicine, 44(5), 701-712.

15. Castellano, J., Casamichana D., y Lago, C. (2012). The Use of Match Statistics that Discriminate Between Successful and Unsuccessful Soccer Teams. Journal of Human Kinetics, 31, 99-106.

16. Cormack, S. J., Newton, R. U. y McGuigan, M. R. (2008), Neuromuscular and endocrine responses of elite players to an Australian Rules Football match. International Journal of Sports Physiology and Performance, 3, 359-374.

17. Currell, K. y Jeukendrup, A. E. (2008). Validity, reliability and sensitivity of measures of sporting performance. Sports Medicine, 38(4), 297-316.

18. Dellal, A., Chamari, K., Wong, D. P., Ahmaidi, S., Keller, D., Barros, R., Bisciotti, G.N. \& Carling, C. (2011). Comparison of physical and technical performance in European soccer match-play: FA Premier League and La Liga. European Journal of Sports Science, 11, 51-59.

19. Dellal, A., Lago, C., Rey, E., Chamari, K., y Orhant, E. (2013). The effects of a congested fixture period on physical performance, technical activity and injury rate during matches in a professional soccer team. British Journal of Sports Medicine. 10.1136/ bjsports-2012-091290.

20. Di Salvo, V., Baron, R., Tschan, H., Calderon, F. J., Bachl, N., y Pigozzi, F. (2007). Performance characteristics according to playing position in elite soccer. Sports Medicine, 28, 222-227.

21. Di Salvo, V., Collins, A., Macneill, B., y Cardinale, M. (2006). Validation of Prozone: A new video-based performance analysis system. International Journal of Performance Analysis in Sport, 6, 108-119.

22. Gregson, W., Drust, B, Atkinson, G., y Salvo V. D. (2010). Matchto-match variability of high-speed activities in premier league soccer. International Journal and Sports Medicine, 31(4), 237-42.

23. Lago, C. (2009). The influence of match location, quality of opposition, and match status on possession strategies in professional association football. Journal of Sports Sciences, 27(13), 1463-1469.

24. Mohr, M., Krustrup, P. y Bangsbo, J. (2003). Match performance of high standard soccer players with special reference to development of fatigue. Journal of Sports Sciences, 21, 519-528.

25. Morgans, R., Adams, D., Mullen, R., y Williams, M. D. (2014). Changes in physical performance variables in an English Championship League team across the competitive season: the effect of possession. International Journal of Performance Analysis in Sport, 14, 493-503.

26. O'Donoghue, P. G. y Tenga, A. (2001). The effect of score-line on work rate in elite soccer. Journal of Sports Science, 19, 25-26. 
27. Rampinini, E., Coutts, A., Castagna C., Sassi, R. y Imperizelli, F. M. (2007). Variation in top level soccer match performance. International Journal of Sports Medicine, 28(12):1018-1024.

28. Rampinini, E., Impellizzeri, F. M., Castagna, C., Coutts, A. J., y Wisloff, U. (2009). Technical performance during soccer matches of the Italian Serie A league: Effect of fatigue and competitive level. Journal of Science and Medicine in Sport, 12(1), 227-233.

29. Rienzi, E., Drust, B., Reilly, T., Carter, J. E. y Martin, A. (2000), Investigation of anthropometric and work rate profiles of elite South
American international soccer players. Journal of Sports Medicine and Physical Fitness, 40, 162-169.

30. SAS Institute Inc. SAS/STAT (1999). Users Guide, Version 8, Chapter 41. Cary, NC: SAS Institute Inc.

31. Taylor, J. B., Mellalieu, S. D., James, N., y Shearer, D. A. (2008). The influence of match location, quality of opposition, and match status on technical performance in professional association football. Journal of Sports Sciences, 26, $885-895$. 